
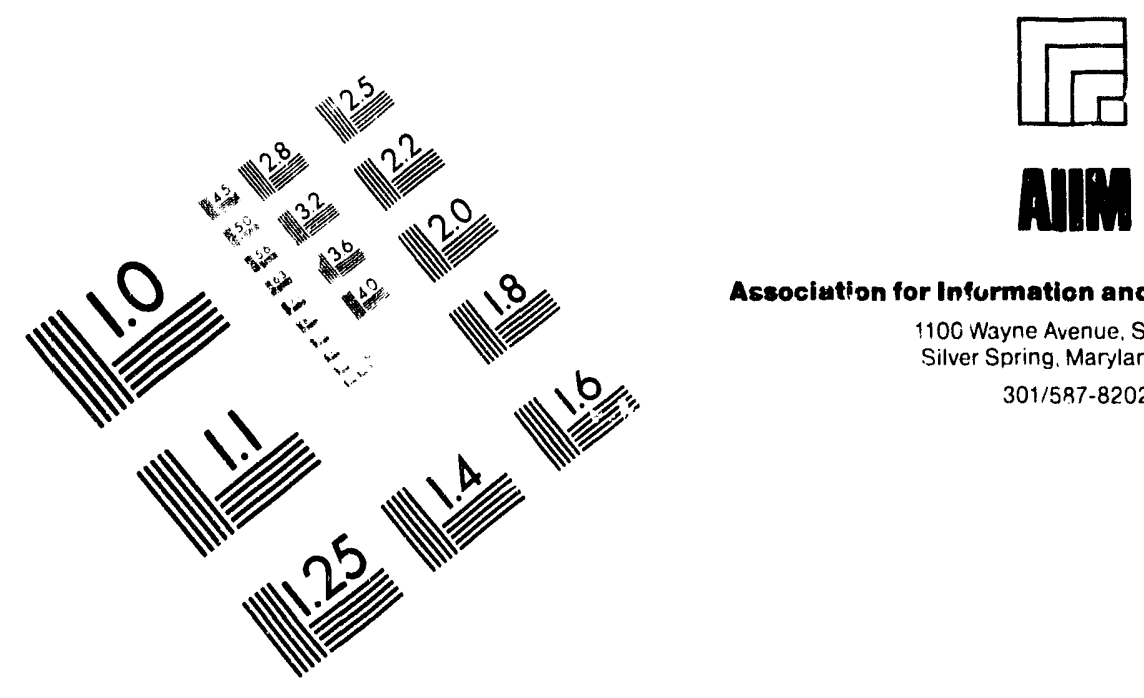

Association for Infurmation and Image Management

1100 Wayne Avenue. Suite 1100

Silver Spring, Maryland 20910

301/587-8202

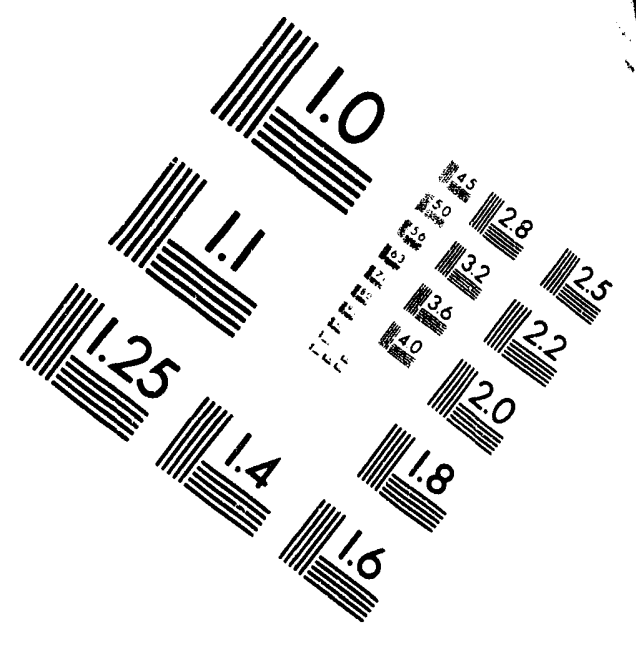

Centimeter

1
2 Inches
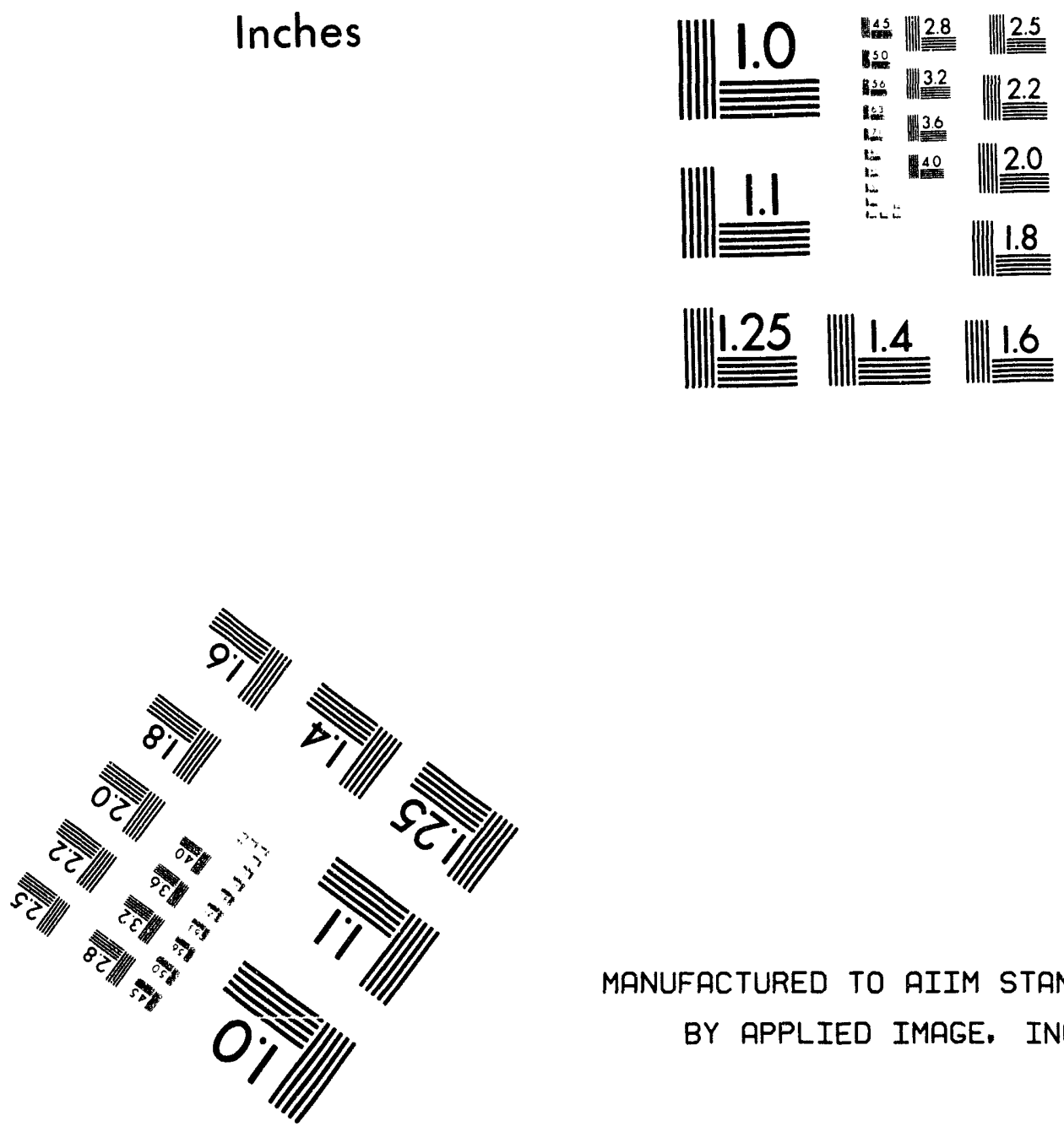

MANUFACTURED TO AIIM STANDARDS

BY APPLIED IMAGE, INC.

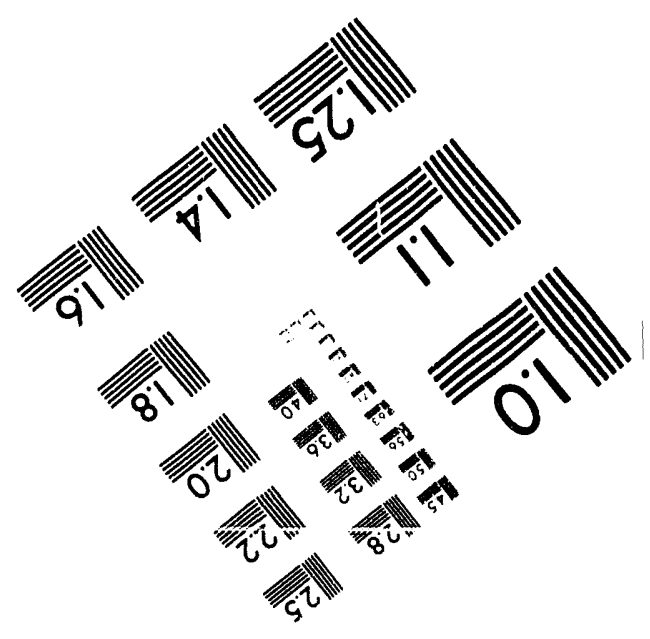



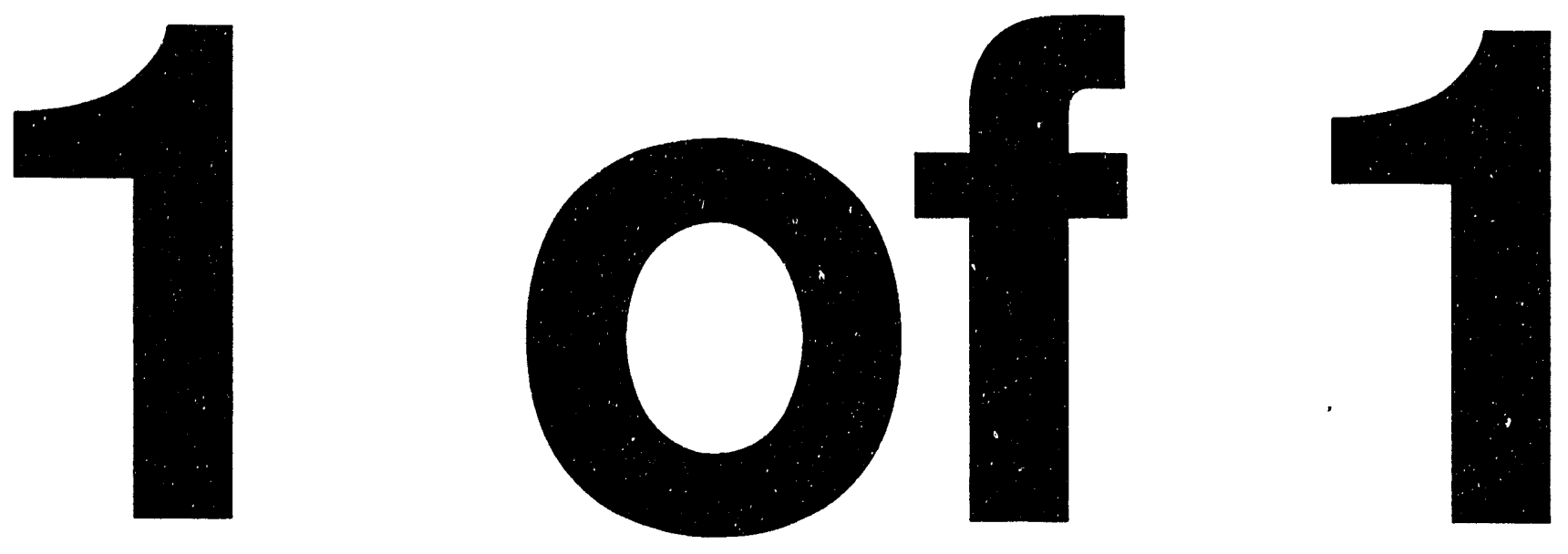


\title{
An Engineering Study and Concept Design of the Supervisory Control and Data Acquisition (SCADA) for Conventional Systems*
}

\author{
L. Norman \\ Superconducting Super Collider Laboratory ${ }^{\dagger}$ \\ 2550 Beckleymeade Ave. \\ Dallas, TX 75237
}

May 1993

*Presented at the Fifth Annual International Symposium on the Super Collider, May 6-8, 1993 San Francisco, CA. ${ }^{\dagger}$ Operated by the Universities Research Association, Inc., for the U.S. Department of Energ." under Contract No. DE-AC35-89ER40486. 


\title{
AN ENGINEERING STUDY AND CONCEPT DESIGN OF THE SUPERVISORY CONTROL AND DATA ACQUISITION (SCADA) FOR CONVENTIONAL SYSTEMS
}

\author{
Leonard S. Norman \\ Engineering and Design Group \\ Conventional Construction Division \\ Superconducting Super Collider Laboratory* \\ 2550 Beckleymeade Avenue \\ Dallas, Texas 75237
}

\begin{abstract}
The study objective was to evaluate several conventional equipment SCADA system architectural concepts and to recommend an approach for development. Each of the concepts given consideration had to satisfy the Superconducting Super Collider (SSC) conventional equipment SCADA application requirements and the evaluation process determined which approach represented the best technical and most cost effective solution to the system requirements. Based on the results of the concept evaluation process, a personal computer based approach was recommended for the SSC conventional equipment SCADA application. Block diagrams and budgetary cost estimate for this approach were developed with specific recommendations with respect to the conventional equipment SCADA system architecture and development process.
\end{abstract}

\section{INTRODUCTION}

This paper discusses the study used to evaluate and recommend a system architecture and concept design for a Supervisory Control and Data Acquisition (SCADA) system for conventional systems of the SSC. Conventional systems are defined as all utility systems such as power distribution, potable water, gas, and HVAC systems. Technical systems such as the low conductivity water, cryogenics and accelerator control systems have separate control systems.

The study evaluated several conventional SCADA system architectural and concept designs and recommended an approach for development. Each concept under consideration during the study had to satisfy SSC conventional systems SCADA application requirements. The evaluation process determined which approach presented the best technical benefits and most cost effective solution to system requirements.

The technical systems will be supported by a high speed networked control system with operations from a central location. The conventional systems SCADA will also have

\footnotetext{
*Operated by the Universities Research Association, Inc., for the U. S. Department of Energy under Contract No. DE-AC35-89ER40486.
} 
centralized monitoring and control with an interface for data exchange between technical and conventional systems.

\section{SYSTEM REQUIREMENTS}

The SSC is a large and complicated facility. The collider tunnel ring, will be over 54 miles in circumference. There will be 10 service areas (spaced approximately 5.4 miles apart) that will be for power distribution, technical systems equipment, ventilation, and surface support equipment to the collider ring. Total input/output ( $/ / O)$ point count for the collider ring has been estimated for conventional systems requirements at 5,000 points.

The injector facilities will consist of a Linear Accelerator (LINAC), a Low Energy Booster (LEB) ring, a Medium Energy Booster (MEB) ring, a High Energy Booster (HEB) ring, and four experiment sites located in areas designated as the east and west campuses. The total $\mathrm{V} / \mathrm{O}$ point count for these facilities will add about another 5,000 points.

The SCADA system has to provide immediate access to status and control data on all utilities, including alarms and problem conditions, with facilities to provide reports of trends, utility usage, maintenance requirements, and archiving.

Flexibility and-state-of-the-art equipment will be required in the system hardware and software, with the ability to adopt upgrades in the future. The system must be able to include:

- A control system that fits into the construction schedule, which reaches completion in the year 1999 or later.

- A system that runs in parallel with the high speed technical network with minimal involvement from the technical operations center.

- The most cost effective system that satisfies all conventional SCADA operational requirements and maintenance requirements.

- A technology that will carry conventional systems SCADA past 1999 and into the foreseeable future.

\section{STUDY OBJECTIVE AND RESULTS}

The study objective was to evaluate conventional SCADA system architectural concepts and to recommend an approach for development. Each of the concepts under consideration must satisfy the SSC conventional equipment SCADA application requirements. The evaluation process had to determine which approach represented the best technical benefit and most cost effective solution to the system requirements.

The information gathered during the study was used to formulate four conventional equipment SCADA system architectural concepts. Each of the concepts considered in the evaluation process was a system configured around commercially available equipment technologies. They were configured to support 30 SSC local sites with 750 data parameters being produced at each site. The actual local site estimate is: collider service areas (10 locations), experimental sites (four locations), main power substation (two locations), and various sites in the injector and campus (four locations), for a total of 20 sites with an I/O count of 10,000 points.

\section{ABBREVIATED CONCEPT DESCRIPTION:}

- CONCEPT A - An off-the-shelf distributed control system (such as Westinghouse, or Foxboro) supporting SCADA master units at each SSC local site.

- CONCEPT B .- Utilized off-the-shelf open architecture computers (such as IBM, DEC, or HP) as the SCADA host and site computers at each local site.

- CONCEPT C - Utilized off-the-shelf VMEbus crate computers at the central control area and at each local site. One of the VMEbus crates at each local site would act as a site master system.

- CONCEPT D - Utilized off-the-shelf IBM format personal computers as the SCADA host and site computers at each local site. 
Concept evaluations were performed using a weighted comparison technique. The weighted evaluation method yields numerical values representing objective and subjective desirability. The objective desirability measures the concept life cycle costs. The subjective desirability measures are the key issues identified in the system requirements.

Based on the results of the concept evaluation process, Concept D was recommended, with additional specific recommendations with respect to the system architecture and development process as follows:

- Hardware should be standard state-of-the-art personal computers.

- Software should be UNIX based or the same software as used by technical systems controls.

- The recommended network for interconnection of site master computers and the host computer is an Ethernet bus conforming to IEEE 802.3 standards. ${ }^{1}$

- A single source for equipment should be specified.

- Acceptable interfaces for technical systems control interconnection with SCADA master computers should be specified.

\section{COICEPT DESIGN}

Concept D, as amended, is shown as a block diagram in Figure 1. The concept SCADA system includes PCs in the central control area and PCs at each of the remote sites. Shared memory will be used at each location for the hot standby and graceful system degradation capabilities needed to support desired system reliability. SCADA system software is based on a QNX (PC version of UNIX) operating system. These choices, coupled with rich market availability of PC hardware/software, will satisfy the concerns of system flexibility and the potential for future upgrades.

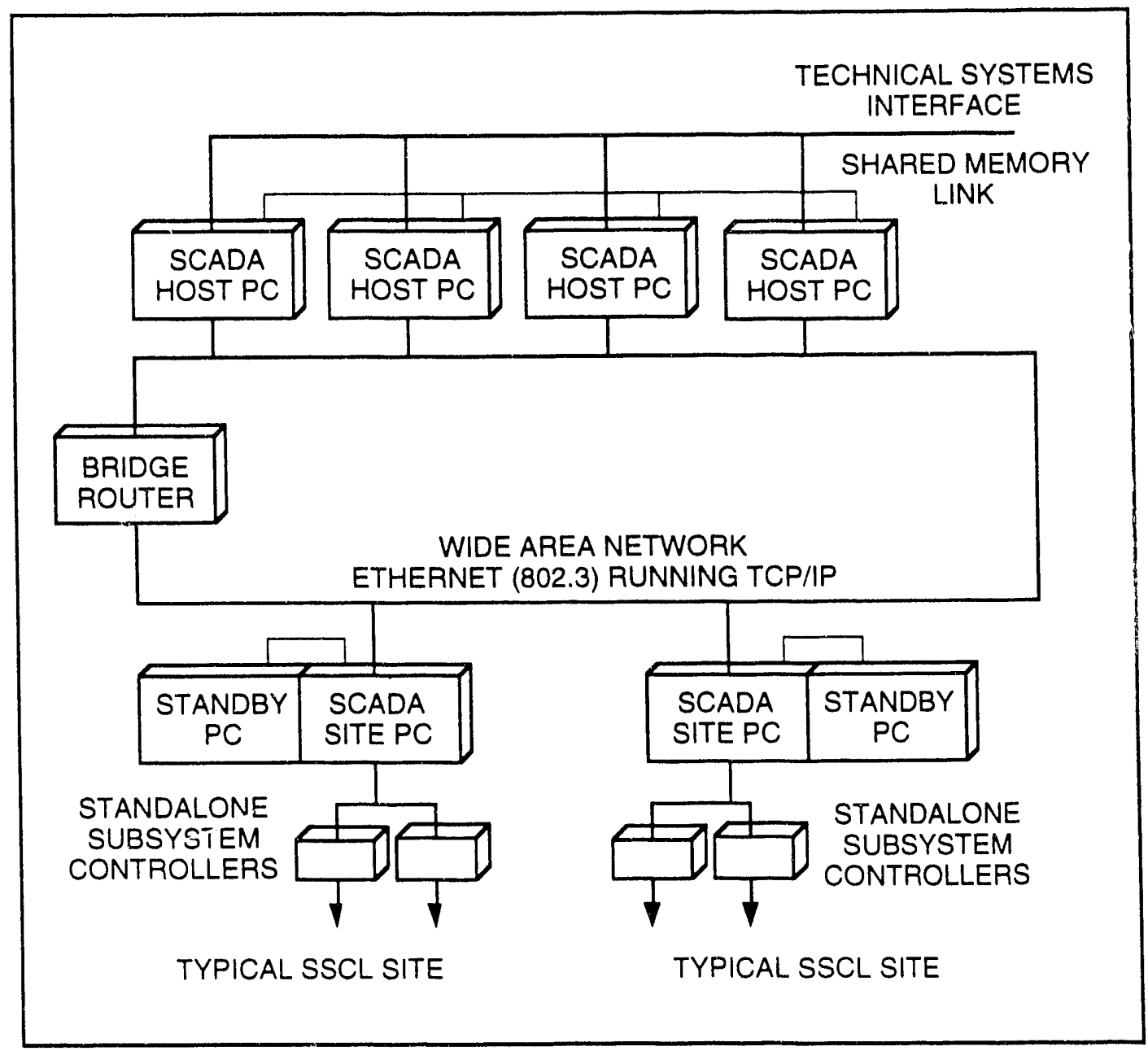

Figure 1. Recommended concept design. 
The communications network between the central control area host Prs and the site PCs will be an Ethernet bus conforming to IEEE 802.3 and other related standards. Ethernet is widely supported in the marketplace. Open standards are available for communications (TCP/IP) on an Ethernet network. The medium bandwidth, as specified by the standard, is 10-MB-per-second on an 18-MHz channel. The Ethernet bus is a broadcast type network that can be implemented in segments. Though Ethernet does not use tokens to sequence through a logical ring, routers are used for addressing nodes to permit central computer polling of the remote sites.

\section{CONCLUSIONS}

The study and concept design demonstrated that a system can be developed to incorporate complex design and construction schedules. As site design details are established, it is anticipated that the conventional SCADA system will undergo changes in system requirements definition. The need to develop interim central control capabilities may be required. To ensure an engineered rather than evolutionary system design, the SCADA system concept design must be implemented as a standardized system with standard equipment configurations.

\section{ACKNOWLEDGMENTS}

The author wishes to recognize and thank James E. Whiteford of Sverdrup Technology Inc. Tullahoma, Tennessee, the Senior Engineer in the development of the study and concept design.

\section{REFERENCES}

1. IEEE. 1990. "Carrier Sense Multiple Access/Collision Detection (CSMA/CD)" Standard 802.3 The Institute for Electrical and Electronic Engineers (IEEE) 802 Standards Committee, New York, NY. 

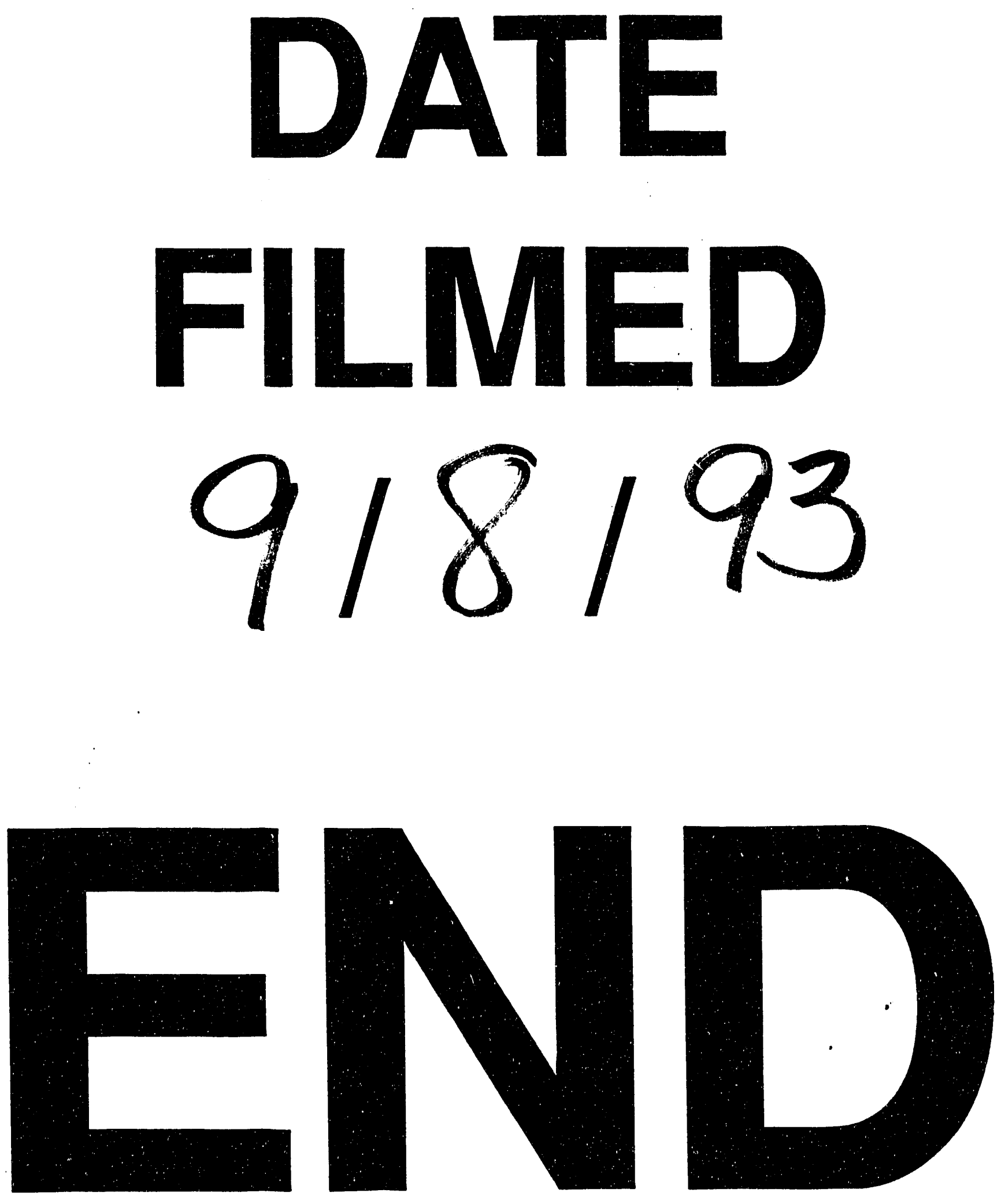
